\title{
The Origin of Millisecond Pulsars
}

\author{
Dipankar Bhattacharya \\ Raman Research Institute, Bangalore 560080, INDIA
}

\begin{abstract}
According to the standard model, millisecond pulsars are the descendants of low-mass X-ray binaries (LMXB). The importance of this formation route has, however, been questioned by several authors on different grounds. This paper critically reviews the arguments and assumptions underlying the standard model. The kinematic properties of the LMXB and millisecond pulsar populations are compared, and are found to be compatible. This provides an additional argument in favour of the standard model.
\end{abstract}

\section{Introduction}

Millisecond pulsars distinguish themselves from the rest of the pulsar population by their rapid spin ( $\sim$ a few ms) and low magnetic field strength $\left(\sim 10^{8}-10^{9} \mathrm{G}\right.$, as opposed to $\sim 10^{12} \mathrm{G}$ for ordinary pulsars). In the field-period distribution for pulsars (fig. 1) they occupy the lower left hand corner, far removed from the majority of pulsars at the upper right.

The special properties of this class of pulsars clearly arise from the fact that they have been members of interacting binary systems. Most of the millisecond pulsars have binary companions, which are usually low-mass degenerate dwarfs. A few of the millisecond pulsars are solitary, but it is the widely held view that these pulsars have been members of binary systems, but the companion has been lost in some way.

Another interesting fact about millisecond pulsars is that they occur in large numbers in globular clusters. This again highlights the importance of interacting binaries in the formation of millisecond pulsars, because globular clusters are fertile grounds for the formation of binaries via stellar encounters. In this paper, however, I will not discuss the rich formation scenarios specific to globular clusters (see Hut (1996) for a recent review), but confine myself to the millisecond pulsars in the galactic disc, where stellar encounters are negligible.

The standard model for the formation of millisecond pulsars rests on three main pillars: 1) that the millisecond pulsars are recycled, i.e. they obtained their rapid spin due to accretion-driven spin-up; 2) that the neutron stars originally had field strengths as strong as that of the majority of pulsars, namely $\sim 10^{12} \mathrm{G}$, which eventually decayed by 3-4 orders of magnitude to their present values; and 3 ) that the progenitors of millisecond pulsars are interacting binary systems similar to the low-mass X-ray binaries, a prominent class of X-ray binaries in which a neutron star accretes matter from $\mathrm{a} \sim 1 M_{\odot}$ donor. I will discuss each of these points in more detail. below. 


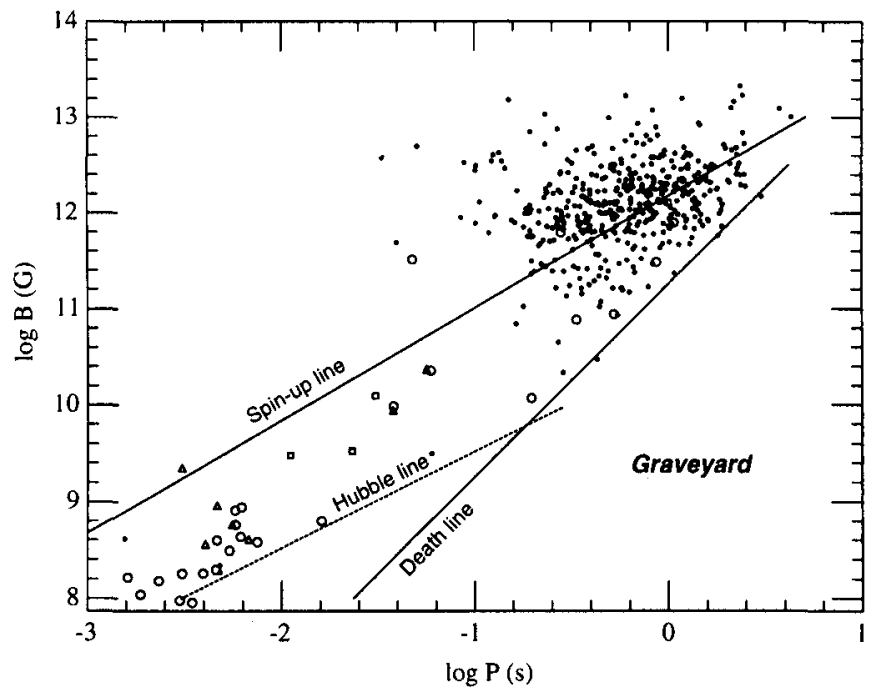

Figure 1. The magnetic fields and periods of known pulsars (see Camilo, this volume for an update). Filled dots indicate isolated pul sars and open circles binaries, both in the galactic disc. Isolated pulsars in globular clusters are shown as triangles and binaries in globular clusters as squares. The spin-up line shows the minimum period to which a neutron star can be spun-up in Eddington-limited accretion. Pulsar activity ceases to the right of the death line, and the spindown age of a pulsar equals $10^{10} \mathrm{y}$ on the Hubble line.

\section{Recycling}

That the rapid spin of millisecond pulsars may be a result of spin-up due to accretion was conjectured right after the discovery of the first millisecond pulsar B1937 +21 , which was not even a member of a binary system (Radhakrishnan and Srinivasan 1982). The spin-up process (also called recycling) can achieve a very short spin period if the stellar magnetic field is low, and the accretion rate is high:

$$
P_{\text {eq }} \simeq 2 \mathrm{~ms}\left(\frac{B}{10^{9} \mathrm{G}}\right)^{6 / 7}\left(\frac{\dot{M}}{\dot{M}_{\text {Edd }}}\right)^{-3 / 7}
$$

being the equilibrium spin period for dipole field strength $B$ at the surface of the neutron star and accretion rate $\dot{M}$ on it; $\dot{M}_{\text {Edd }}$ is the Eddington accretion rate (see Bhattacharya and van den Heuvel 1991 for a review).

The ärguments in favour of millisecond pulsars being recycled include the following:

- A large fraction of millisecond pulsars (in the galactic disc) do have binary companions at present, and the nature of these companions (low-mass white dwarfs) indicate that mass transfer must have taken place. 
- The low orbital eccentrities are also suggestive of a prolonged mass transfer phase. Soon after the formation of the neutron star in a supernova, one expects the orbit to have acquired a large eccentricity. The reduction of these eccentricities to their present values $\left(\ll 10^{-3}\right)$ requires a long period of tidal contact, with the donor star nearly filling the Roche lobe (see Phinney 1992 for a discussion).

- Ongoing mass transfer in many accretion-powered X-ray pulsars appears to lead to a spin-up of the neutron star (see, e.g. Nagase 1989). Mass transfer in the progenitor systems of millisecond pulsars is expected to have had a similar effect.

- The period-magnetic field combination of recycled pulsars are expected to be confined to the right of the "spin-up line" in fig. 1 . As we see, practically all millisecond pulsars do indeed fall in this region.

The above list shows that the evidence for millisecond pulsars being spun-up is fairly strong, but is entirely circumstantial. There is no direct evidence that in systems capable of producing final orbital characteristics like millisecond pulsars such a spin-up is indeed going on. In the most promising progenitor candidates, the Low-Mass X-ray Binaries, the spin rate of the neutron stars have so far not been observed, let alone the rate of change of spin. A direct measurement of these quantities will be the final test of the recycling hypothesis of millisecond pulsar origin.

\section{Magnetic field decay}

The magnetic field strengths of all millisecond pulsars are low $\left(\sim 10^{8}-10^{9} \mathrm{G}\right)$, and in fact this is necessary for the spin-up process to achieve periods in the millisecond range. According to the standard picture, these low field strengths are supposed to come about by a decay of the original field the neutron star had $\left(\sim 10^{12} \mathrm{G}\right)$. There is, however, no convincing evidence that magnetic fields of neutron stars decay spontaneously. Recent population synthesis studies (Bhattacharya et al 1992; Wakatsuki et al 1992; Hartman et al, this volume) find no evidence of field decay among isolated pulsar population. Even among recycled pulsars the cases where independent age estimates have been possible, mainly by optical studies of their companions, show that their present field strengths are stable over long times (see e.g. van Kerkwijk, this volume). Significant spontaneous field decay due to ohmic processes in a star of such high conductivity as a neutron star is difficult to understand even from a theoretical point of view (Baym, Pethick \& Pines 1969).

Because of these reasons, the widely held view at present is that the magnetic field of a neutron star decays mainly due to processing in a binary system (Bailes 1989). There is no consensus as yet on the exact physical mechanism of this, and suggestions abound. The field reduction could be due to flux expulsion from the interior as a result of pre-recycling spin-down (Srinivasan et al 1990; Jahan Miri and Bhattacharya 1994), or enhanced Ohmic decay due to accretioninduced heating of the stellar crust (Geppert \& Urpin 1994; Urpin \& Geppert 1995), or even flux-dragging by the accreted material, reconnection and burying 


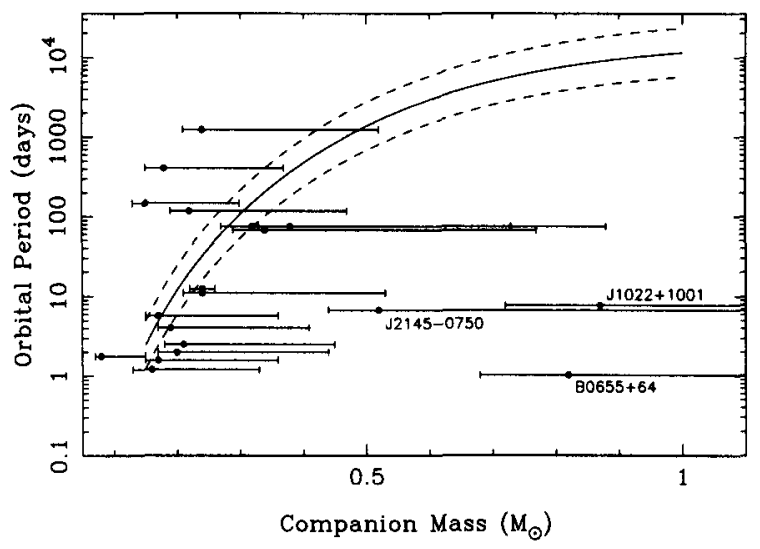

Figure 2. Orbital period-companion mass relation of pulsars with white dwarf companions in (very nearly) circular orbits, compared with the theoretical relation of Joss, Rappaport and Lewis (1987) (solid curve). The dashed lines represent a factor-of-two uncertainty in the theoretical relation. The three pulsars with names indicated, i.e. J1022+1001, J2145-0750 and B0655+64 are likely products of intermediate-mass binaries, while the rest are products of low-mass systems. The error bars correspond to 10 to $90 \%$ probability range for orbital inclinations. After Lorimer (1994).

under the surface (Bisnovatyi-Kogan and Komberg 1974; Romani 1990). More work is necessary to settle this question.

Whatever be the actual mechanism of the decay of the magnetic field, the fact remains that in all classes of neutron stars studied, it appears that their present fields are steady and long-lived. So the field decay, if it occurs, must happen in a phase during which the neutron star is either invisible or unrecognisable, or its field is unmeasurable by present means.

\section{Progenitors}

Of the three pillars mentioned above the third, namely, the issue of the progenitors of millisecond pulsars, has received, and continues to receive the greatest amount of attention in the literature. On the one hand, the characteristics of millisecond pulsars point distinctly to evolution in binary systems with low-mass donors. The arguments for this are:

- Spinning-up to millisecond periods requires accretion of $\sim 0.1 M_{\odot}$ of matter, and therefore a prolonged phase of heavy accretion $\left(z 10^{7} \mathrm{y}\right.$ at Eddington rate). Stable Roche-lobe overflow for such a long duration is possible only if the donor star has a mass nearly equal to, or lower than the mass of the neutron star, similar to known low-mass X-ray binaries (LMXBs).

- The present-day companions of millisecond pulsars are mainly low-mass white dwarfs (mass $\leqslant 0.3 M_{\odot}$ ). These objects can form only from low-mass 
stars, and that too if significant amount of envelope matter is removed during evolution. These remnants are therefore consistent with the products expected in low-mass X-ray binaries.

- The final products of wide LMXBs (initial orbital periods longer than $\sim 1$ day) are expected to follow a relation between the orbital period and the mass of the degenerate dwarf remnant, since the radius of the donor (which would have to be evolved in such wide orbits) is determined almost exclusively by the mass of its helium core till the time of detachment. Figure 2 shows the expected orbital period-companion mass relation according to Joss, Rappaport and Lewis (1987), along with the positions of a number of known pulsars with white dwarf companions. Barring J1022+1001, $\mathrm{J} 2145-0750$ and $\mathrm{B} 0655+64$, all of which are likely remnants of intermediate mass binaries, the remaining objects are seen to more or less follow the expected relation, strenghtening the argument in favour of their origin in LMXB-like objects. Of late, however, there has been some discussion as to whether significant deviation from the above relation might occur due to strong angular momentum loss and/or mass loss from the system (see Tauris, this volume; Ergma, this volume as well as the debate contributions following this paper).

While the above suggests that LMXBs may provide comfortable breeding grounds for millisecond pulsars, this hypothesis is in fact hotly debated in the literature. The strongest arguments against origin from LMXBs have been the following:

- The birth rate of observed LMXBs has been estimated to be one to two order of magnitudes lower than that of millisecond pulsars (Kulkarni and Narayan 1988). More recent estimates (e.g. Lorimer 1995) conclude that there may be a overall agreement between the net birthrate of LMXBs and that of millisecond pulsars, but if one looks at the short orbital period $(\lessgtr 20 \mathrm{~d})$ and long orbital period $(~(20 \mathrm{~d})$ millisecond pulsar systems separately, then the discrepancy persists for the short-period class.

- Many observed low-mass X-ray binaries have orbital periods less than 10 hours. These binaries are expected to evolve via angular momentum loss to shorter orbital periods. Among the observed millisecond pulsars very few have orbital periods as short as that. Therefore a major fraction of known low-mass X-ray binaries will not lead to the observed type of binary millisecond pulsars.

- Among the millisecond pulsars in the galactic disc a few are single. The origin of such single millisecond pulsars remains poorly understood, and it is not certain that their formation routes involve standard low-mass X-ray binaries.

- If LMXBs harbor fast-spinning neutron stars, one hopes that the rapid spin of the neutron star should become visible in some manner. In the $\mathrm{X}$-ray phase, no pulsation has yet been detected - perhaps due to poor collimation of the accretion flow and/or the presence of a shrouding corona. But once accretion ceases one expects the rapidly spinning neutron star to make its appearance as a pulsar. Searches in quiescent LMXBs, in the long 
dormant phase between mass transfer episodes, have however not revealed the presence of any millisecond pulsars (Kulkarni et al 1992).

There have been a number of attempts to address the birthrate problem mentioned above. One way to make the birth rates match would be to reduce the effective X-ray life times of LMXBs, which are normally estimated to be of order $10^{8}-10^{9} \mathrm{y}$. While life times much shorter than this may not be applicable to most of the observed systems, there might be a class of short-lived LMXBs with a large birth rate, poorly represented in the observed sample because of its limited life span. One promising suggestion in this direction is due to van den Heuvel (1995): binaries containing donors of mass between 1.4 and $2.3 M_{0}$ would have an unstable mass transfer to start with, but this will be followed by a short $\left(\sim 10^{7} \mathrm{y}\right)$ period of stable mass transfer. In the unstable phase there would be little accretion onto the neutron star, and no visible $\mathrm{X}$-rays, and in the stable phase the system would look very much like a standard LMXB. This duration of mass transfer is however adequate for spinning a neutron star up to a millisecond period, and the products are expected to follow the companion mass-orbital period relation appropriate for LMXBs.

Other suggested mechanisms for shortening LMXB lifetimes include the arresting of accretion flow by the pulsar wind as soon as spin-up to a few millisecond period has been achieved (Ruderman, Shaham and Tavani 1989; Bhattacharya and Srinivasan 1989), and a short-lived accretion (but long enough for spin-up) from the debris of a secondary disrupted when the newly born neutron star plunged into it as a result of a kick received in a supernova explosion (Phinney and Hansen 1993). Obviously, the last scenario is applicable only to the formation of isolated millisecond pulsars, or perhaps those with planetary companions.

A radically different suggestion has been that millisecond pulsars result from accretion-induced collapse (AIC) of white dwarfs, which form low-field, fast-spinning neutron stars directly without a subsequent LMXB phase (Michel 1987; Grindlay and Bailyn 1988; Bailyn and Grindlay 1990). This is at best a conjecture at present, and many questions, such as why the resulting neutron star should have a low field, why should there be no accretion after AIC, and whether this channel can provide enough birth rate remain open.

\section{Kinematics of LMXBs and millisecond pulsars}

If millisecond pulsars are indeed the descendants of LMXBs, the kinematic properties of these two populations should be consistent with each other. This provides an additional test of the evolutionary link. In an attempt to check this consistency, we (Ramachandran and Bhattacharya 1996) have performed a population synthesis study as described below.

The kinematic properties of a population include the velocity distribution and the spatial distribution in the galaxy. In case of LMXBs, the number of reliable velocity measurements are few, but we have a fair idea of their spatial distribution, as LMXBs, being powerful X-ray sources, can be observed at large distances. The observed population of millisecond pulsars, however, is restricted to the immediate solar neighbourhood, so their spatial distribution is poorly known. On the other hand, reliable measurements of velocities transverse to the 

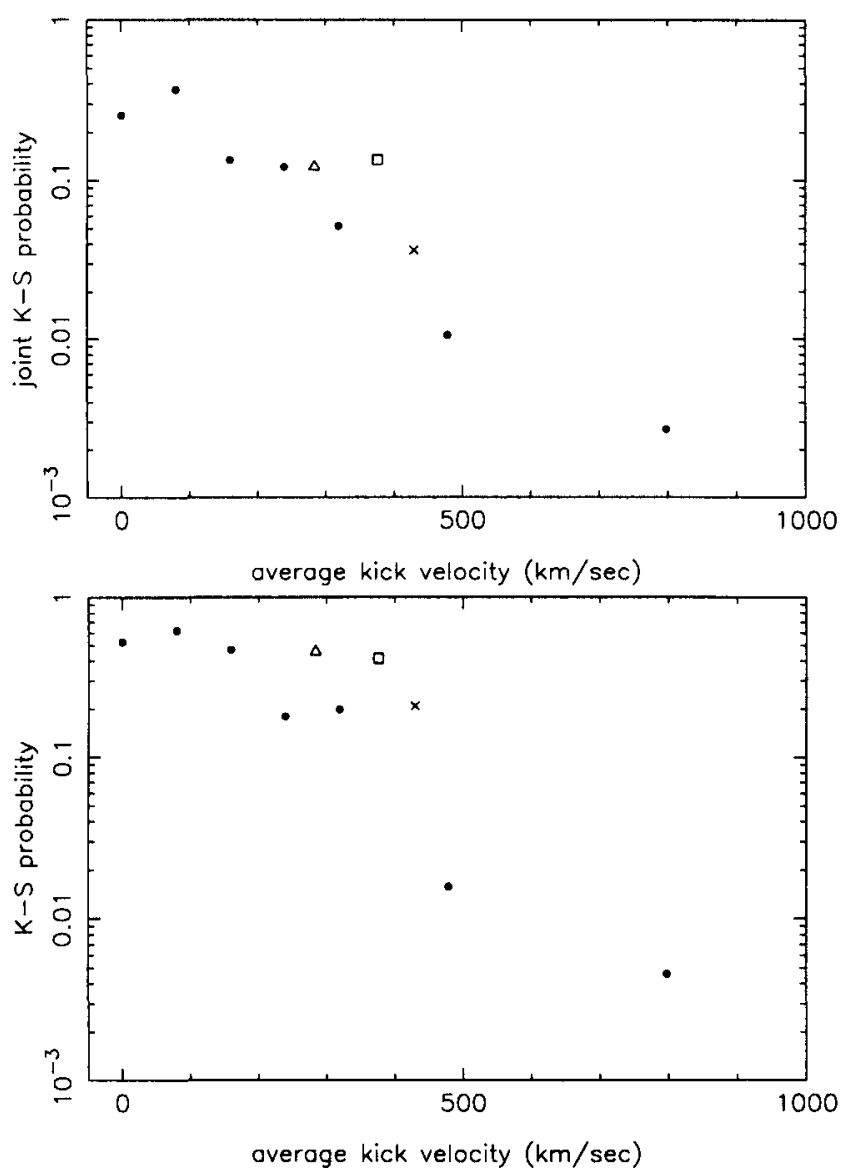

Figure 3. The Kolmogorov-Smirnov fit probabilities to the spatial distribution of LMXBs (upper panel) and velocity distribution of millisecond pulsars (lower panel) of synthetic populations generated with different assumed kick speed distributions. The dots indicate Maxwellian distributions of various widths, the cross represents the Lyne-Lorimer distribution, the triangle the Paczyński (1990) distribution, and the square the Phinney (1994) modification of the same. 
line-of-sight are available for at least 12 millisecond pulsars (Nice and Taylor 1995; Nicastro and Johnston 1995; Camilo et al 1994).

In our study, we generate a simulated population of binaries, with $\geq 10 M_{\odot}$ primaries and $\leq 1.5 M_{\odot}$ secondaries, evolve each system through the spiral-in of the secondary into the primary, and eventually the explosion of the primary resulting in the formation of a neutron star. We assume various distributions of the kick speeds received by the neutron stars at birth, including Maxwellians of different widths, the distribution proposed by Lyne and Lorimer (1993), that by Paczyński (1990), and the modification of the Paczyński (1990) law suggested by Phinney (1994). We enumerate the surviving systems, select those which are likely to lead to mass transfer in a Hubble time, evolve their orbits in the Galaxy, and note the final position and velocity after an age in the interval 0 to $10^{10} \mathrm{y}$.

We then compare the resulting space distribution with that of observed LMXBs. For each assumed kick speed distribution we adjust the radial distribution of birth rate in the galaxy to get the best possible fit. We intercompare these best fits for different kick speed distributions.

For each of these best-fit distributions, we select objects that fall into a small heliocentric volume which contains most of the known millisecond pulsars. We then compare the velocity distribution of these subsamples with that of millisecond pulsars.

Fig. 3 shows the comparative fit probabilities for different assumed kick speed distributions. We see that decent fits to the LMXB space distribution, as well as to the velocity distribution of millisecond pulsars, is obtained for Maxwellians with average kick speeds below $200 \mathrm{~km} \mathrm{~s}^{-1}$, and the Paczyński (1990) distribution, as well as its Phinney (1994) modification produce fairly good fits too. Lyne and Lorimer (1993) distribution does not fit so well, but with the limited data at present it is still within the limits of acceptability. The similarity in the pattern between the two panels of fig. 3 is striking, and we may conclude that within the present limits of uncertainty, the kinematic properties of LMXBs and millisecond pulsars appear to be fully consistent.

\section{Summary}

In summary, then, the standard model of the origin of millisecond pulsars appears to fare quite well, provided we are willing to allow that

- Recycling to millisecond period occurs, but is not observable with currently available means.

- Field decay from $\sim 10^{12} \mathrm{G}$ to $\sim 10^{8}-10^{9} \mathrm{G}$ takes place, but does so in a phase that is not observable, and

- Millisécond pulsars do descend from LMXBs, of which a large fraction cannot be seen due to their short life times.

It is also clear that our knowledge in most of the above aspects is rather inadequate, and I would like to conclude with a series of questions that the forthcoming, as well as future debates on the subject are likely to address. 
1. Do we understand LMXB evolution?

- Is there an orbital period - core mass relation?

- How much mass is accreted on the neutron star? At what rate? What is the average active life time of an LMXB?

- What are the end products of LMXBs with short orbital periods?

- Does LMXB evolution produce an orbital period gap in its final remnants? If so, at what range of orbital periods?

2. How do single millisecond pulsars, millisecond pulsars with planetary companions, and pulsars with very low mass $\left(\lesssim 0.01 M_{\odot}\right)$ binary companions form?

3. Is accretion-induced collapse important? Do we need as progenitors of millisecond pulsars systems other than LMXBs?

4. What is the mechanism for magnetic field decay?

5 . What is the origin of pulsars in globular clusters?

- Why is there a large fraction of single pulsars among them?

- What causes the large observed spread in the magnetic field strength and spin period of globular cluster pulsars?

Acknowledgments. I thank the International Astronomical Union and the organisers of the colloquium for travel support and cordial hospitality at Sydney.

\section{References}

Bailes M., 1989, ApJ, 342, 917

Bailyn C. D., Grindlay J. E., 1990, ApJ, 353, 159

Baym G., Pethick C., Pines D., 1969, Nature, 224, 673

Bhattacharya D., Srinivasan, G., 1989, Curr.Sci., 58, 953

Bhattacharya D., van den Heuvel E. P. J., 1991, Phys.Rep., 203, 1

Bhattacharya D., Wijers R. A. M. J., Hartman J. W., Verbunt F., 1992, A\&A, 254,198

Bisnovatyi-Kogan G. S., Komberg B. V., 1974, Soviet Ast., 18, 217

Camilo F., Foster R. S., Wolszczan A., 1994, ApJ, 437, L39

Geppert U., Urpin V., 1994, MNRAS, 271, 490

Grindlay J. E., Bailyn C. D., 1988, Nature, 336, 48

Hut P., 1996, in van Paradijs J., van den Heuvel E. P. J., Kuulkers E., eds, IAU symposium 165: Compact Stars in Binaries, page 377, Kluwer Academic Publishers, Dordrecht

Jahan Miri M., Bhattacharya D., 1994, MNRAS, 269, 455

Joss P. C., Rappaport S. A., Lewis W., 1987, ApJ, 319, 180

Kulkarni S. R., Narayan R., 1988, Ap.J, 335, 755 
Kulkarni S. R., Navarro J., Vasisht G., Tanaka Y., Nagase F., 1992, in van den Heuvel E. P. J., Rappaport S. A., eds, X-ray Binaries and Recycled Pulsars, page 99, Kluwer Academic Publishers, Dordrecht

Lorimer D. R., 1994, Ph.D. Thesis, University of Manchester, UK

Lorimer D. R., 1995, MNRAS, 274, 300

Lyne A. G., Lorimer D. R., 1993, Nature, 369, 127

Michel F. C., 1987, Nature, 329, 310

Nagase F., 1989, PASJ, 41, 1

Nicastro L., Johnston S., 1995, MNRAS, 273, 122

Nice D. J., Taylor J.H., 1995, ApJ, 441, 429

Paczyński B., 1990, ApJ, 348, 485

Phinney E. S., 1992, Phil.Trans.R.Soc.Lond.A, 341, 39

Phinney E. S., 1994, Paper presented at the Diamond Jubilee Symposium on Pulsars, Indian Academy of Sciences, Bangalore, March 14-17

Phinney E. S., Hansen B. M. S., 1993, in Phillips J. A., Thorsett S. E., Kulkarni S. R., eds, Planets Around Pulsars, page 371, Astronomical Society of the Pacific Conference Series

Radhakrishnan V., Srinivasan G., 1982, Curr.Sci., 51, 1096

Ramachandran, R., Bhattacharya, D., 1996, in preparation

Romani R. W., 1990, Nature, 347, 741

Ruderman M., Shaham J., Tavani M., 1989, ApJ, 336, 507

Srinivasan G., Bhattacharya D., Muslimov A. G., Tsygan A. I., 1990, Curr.Sci., 59,31

Urpin V., Geppert U., 1995, MNRAS, 275, 1117

van den Heuvel E. P. J., 1995, J.Astrophys.Astr., 16, 255

Wakatsuki S., Hikita A., Sato N., Itoh N., 1992, ApJ, 392, 628 